\title{
Cardiolab : A Virtual Laboratory for the analysis of Human Circulatory System*
}

\author{
Alher Mauricio Hernandez, Gino Pierfranco Herrera \\ Grupo de Investigación en Bioelectrónica e Ingeniería Clínica (GIBIC) \\ Universidad de Antioquia (UdeA), Medellín, Colombia
}

Miguel Angel Mañanas

Departament d'Enginyeria de Sistemes, Automàtica i Informàtica Industrial (ESAII)

Centre de Recerca en Enginyeria Biomedica (CREB)

CIBER de Bioingeniería,Biomateriales y Nanomedicina (CIBER-BBN), Spain

\author{
Ramon Costa-Castelló \\ Institut d'Organització i Control de Sistemes industrials (IOC) \\ Departament d'Enginyeria de Sistemes, Automàtica i Informàtica Industrial (ESAII) \\ Universitat Politècnica de Catalunya (UPC)
}

\begin{abstract}
One of the career areas included in the field of Biomedical Engineering is the application of engineering system analysis: physiological modelling, simulation and control. This paper describes a Virtual Laboratory for the analysis and the study of Human circulatory system. The Virtual Laboratory is based on the compilation of several mathematical models described in the literature. Presented application has been build using MATLAB/Simulink and EJS, so it combines good computation capabilities and it is completely interactive. The Virtual Laboratory is designed in order to understand the operation of the circulatory system under normal conditions, and to predict circulatory variables at different levels of stimuli and conditions.
\end{abstract}

\section{Introduction}

BioMedical Engineering (BME) is a discipline that advances knowledge in engineering whose principles and techniques are applied to the medical field and clinical practice. It includes the acquisition of new knowledge and understanding of biological systems through the application of experimental and analytical techniques based on the engineering sciences; and the development of new devices, algorithms, processes and systems that advance

\footnotetext{
* This work were partially supported by Proyecto de Cooperación Internacional (CCD-U013) the Spanish government (DPI2007-62582, TEC2008-02754), and the AGAUR (2007MQD00046) projects. We thank Robinson Montes and Luis Alfonso Vásquez for their collaboration
}

biology and medicine and improve medical practice and health care diagnosis and treatment.

Because of the interdisciplinary nature of this activity, there is considerable interplay and overlapping of interest and effort between engineering and biologic points of view. A tool that has been proved to be efficient in order to shortcut and simplify the access to new concepts and technologies is interactivity $[3,8]$. Interactivity allows to understand qualitatively the influence of parameters in the system behavior without the need of an in-depth knowledge of a certain subject. This is usually the case in BME, where engineers do not need a deep knowledge of certain medical topics (and vice versa). In this work an application developed in order to apply this concept is presented.

$\mathrm{BME}$ is different to other engineering areas in the sense of obtaining results from experimental procedures and reproducing real physiological situations. It is very difficult and expensive to interact with the human being body (collaboration of volunteers or patients to be analyzed, instrumentation with specials safety conditions, ethic and legal requirements for the protocols, etc) and even dangerous in certain situations. The use of Virtual Labs is proposed in order to overcome this drawback and allow students and researchers to interact with the human body. These labs can be built in a complete interactive way, so the students can easily understand the qualitative behavior behind the complex models used to represent the human body.

The field of BME now includes many career areas, and one of them is the application of engineering system analysis (physiologic modeling, simulation, and control) to biologic problems [1]. The case selected in this work is the computer modeling of a physiologic system in order to un- 
derstand its operation under normal situations and to predict physiological variables at different levels of stimuli and conditions.

The cardiovascular system is composed mainly of the blood, heart, and blood vessels. Blood circulates throughout the body to provide individual cells with oxygen and nutrients and helps dispose of metabolic wastes. Arterial pressure control is carried out by a sophisticated non-linear multi-input, multifeedback system termed the baroreflex [13]. The Autonomic Nervous System (ANS) interacts with the cardiovascular system. This interaction and stimuli from the former is critical to understand the function of the latter and can provide significant prognostic information. The ANS is predominantly an efferent system transmitting impulses from the Central Nervous System (CNS) to peripheral organs. Its effects include control of heart rate and force of heart contraction, constriction and dilatation of blood vessels, contraction and relaxation of smooth muscle in various organs, and glandular secretions. Autonomic nerves constitute all of the efferent fibers that leave the CNS, except for those that innervate skeletal muscle. There are some afferent autonomic fibers (ie, from the periphery to the CNS) that innervate the baroreceptors and chemoreceptors in the carotid sinus and aortic arch, which are important in the control of heart rate, blood pressure, and respiratory activity [6].

The Virtual Laboratory presented in this paper is a useful tool for understanding the cardiovascular system operation, for identifying the stimuli from the autonomic nerve system and for predicting different situations in a visually attractive and interactive way for the student and researcher on BME.

\section{The Cardiovascular System}

\subsection{Model Description}

\subsubsection{From autonomic control}

Empirical and functional models have been proposed in the literature to describe various aspects of the cardiovascular system. Some of them also include the respiratory system interaction through an autonomic neural controller [7]. The cardiovascular system is structured in different intermediate processes (see Fig. 1). Components in the system include a nonlinear carotid sinus compartment stimulated by the arterial blood pressure, a sino-atrial node component that regulates the heart rate with both parasympathetic and sympathetic controls, a peripheral resistance component controlled by the alphasympathetic activity, a hemodynamic submodel based on a simple two-element Windkessel model, and a component that controls changes in stroke volume via changes in the venous return and cardiac contractility [13].

Important components in the closed-loop cardiovascular system are the cardiovascular reflex models that includes:

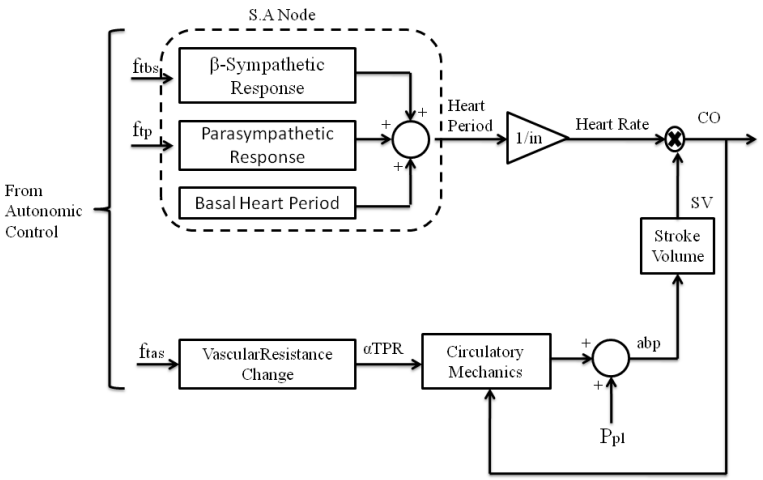

\section{Figure 1. Block diagram of the cardiovascu- lar system described by [13] and [2].}

- baroreflex associated with the pressure receptors that produce both parasympathetic and sympathetic neural activity changes, all represented by the carotid sinus firing frequency, $f_{c s}$ [13], when arterial blood pressure is modified.

- Chemical reflex that is stimulated by both Oxygen and Carbon Dioxide contents modifying the chemoreceptors firing.

- Lung Stretch Receptors that modify their activity, $f_{l s}$, as a function of respiratory tidal volume.

The central autonomic control determines the total $\alpha$ sympathetic, $f_{\text {tas }}, \beta$-sympathetic, $f_{\text {tbs }}$, and parasympathetic, $f_{t p}$, influences on heart rate and peripheral resistance from the baroreflexes, chemoreflexes and lung stretch receptors reflexes. Autonomic control afferent signals, $f_{\text {tas }}, f_{\text {tbs }}$ and $f_{t p}$ allow simulation of different stimuli related with the sympathetic and parasympathetic systems [14].

\subsubsection{Sino Auricular Node.}

Ursino [13] describes the S.A. Node as a subsystem that translates changes in $\beta$-sympathetic and parasympathetic efferent activity into changes in Heart Period, HP (reciprocal of instantaneous heart rate). The heart period level is obtained by assuming a linear interaction between the sympathetic and parasympathetic responses.

$$
H P=\Delta H P_{b s}+\Delta H P_{p}+H P_{b a s a l}
$$

being $\Delta H P_{b s}$ and $\triangle H P_{p}$ the changes in $H P$ which are modulated by $\beta$-sympathetic and parasympathetic responses respectively, and $H P_{b a s a l}$ denotes heart period in the absence of cardiac innervations, that is $0.58 \mathrm{~s}$ in one $70-\mathrm{kg}$ body weight subject. $\beta$-Sympathetic response is 
modelled assuming a first-order dynamics:

$$
\frac{d}{d t} \Delta H P_{b s}=\frac{1}{\tau_{b s}}\left[-\Delta H P_{b s}(t)+\sigma_{b s}(t)\right]
$$

Being $\tau_{b s}$ the $\beta$-sympathetic time-constant, and $\sigma_{b s}$ one function related to $\beta$-sympathetic firing frequency, $f_{t b s}$, that can be considered zero when the firing frequency is too low. Delay associated with the $\beta$-sympathetic effect on the heart period, is longer than that of the parasympathetic response. The parasympathetic or vagal response is also modeled assuming first-order dynamics:

$$
\frac{d}{d t} \Delta H P_{p}=\frac{1}{\tau_{\text {para }}}\left[-\Delta H P_{p}(t)+\sigma_{p}(t)\right]
$$

Similarly, in this case $\tau_{\text {para }}$ is the Parasympathetic time constant and $\sigma_{p}$ is proportional to parasympathetic firing frequency, $f_{t p}$.

\subsubsection{Total Peripheral Resistance (TPR).}

The $\alpha$-sympathetic nerves control the peripheral vascular activities. During hypotension or hypertension, vasoconstriction or vasodilatation occurs to prevent further decreasing or increasing in the blood pressure. This subsystem is modeled using a first-order dynamic system as in the case of the $\beta$-sympathetic component:

$$
\begin{aligned}
\alpha_{T P R} & =\alpha_{P R}+\alpha_{T P R, 0} \\
\frac{d}{d t} \alpha_{P R} & =\frac{1}{\tau_{\text {tas }}}\left[-\alpha_{P R}(t)+\sigma_{P R}(t)\right]
\end{aligned}
$$

Being $\alpha_{T P R}$ the change factor of the total peripheral resistance. $\alpha_{T P R, 0}$ the base $T P R$ change value, $\tau_{\text {tas }}$ the $\alpha$-sympathetic time constant and $\sigma_{P R}$ one function associated with $\alpha$-sympathetic firing frequency, $f_{\text {tas }}$, that can be considered negligible when the firing frequency is too low.

\subsubsection{Stroke Volume and Circulation.}

The stroke volume and the heart period determine the cardiac output, $C O$ (see Fig 1). The circulation helps to carry the nutrients and remove the waste from the body tissues by transportation of blood. Peripheral resistance adjusts the blood flow. Inputs for this subsystem are the heart period, $H P$, the scaling factor for the total peripheral resistance, $\alpha_{T P R}$ and the pleural pressure, $P_{p l}$. An intermediate variable is the arterial blood pressure, $a b p$ and the resulting output variable in the cardiac output, $C O$. Arterial blood pressure is modulated by the vasculature and cardiac output. Resistance and compliance in the circulation are controlled by the $\alpha$-sympathetic nerves. In the model proposed by Cavalcanti and Belardinelli [2], the circulation subsystem used a two-elements Windkessel model where the inputs were the cardiac output and scaling factor for the total peripheral resistance, $\alpha_{T P R}$ such that the model determines the arterial blood pressure, $a b p$, by means of the following expression:

$\frac{d}{d t} a b p(t)=\frac{1}{C_{a r t}\left(\alpha_{T P R} R_{T P R}\right)}\left[\alpha_{T P R} R_{T P R} C O(t)-a b p(t)\right]$

where $C_{\text {art }}$ represents the Arterial Compliance and $R_{T P R}$ the Total Peripheral Resistance.

The stroke volume, $S_{V}$, is determined by the venous return, $V_{n}$, the heart period and the heart contractility, $C_{n}$ [11]. Later has minor effect on the stoke volume:

$$
S V=H P \cdot V_{n}+C_{n}
$$

Venous return and contractility are first order functions with $a b p$ as input variable and with their respective delays.

\subsection{Cardiovascular Stimuli}

Almost every process that affects the autonomic control system also affects the cardiovascular system through the vagal and sympathetic activity. Five stimuli are considered in Cardiolab.

The Autonomic Nervous System plays an important role in the regulation of cardiac adaptation during dynamic exercise and during the exposition to certain substances in humans and conscious animals. For instance, Heart rate $(H R)$ and stroke volume $(S V)$ increase during rhythmic or dynamic exercise, which produces an increase in cardiac output. Recent works stated that cardiac sympathetic nerve discharge, $f_{t b s}$, increases during exercise in proportion to the running speed, for instance and that cardiac sympathetic outflow is stimulated during dynamic exercise although the intensity of exercise is moderate and even low, in concert with cardiac parasympathetic withdrawal [12].

The cholinergic intoxication is considered a stimulus of the cardiovascular system due to the increase of parasympathetic system activity and produces also an increase of the cardiac period (bradycardia) and hypotension. It is known that this kind of intoxication can be produced by the contact of respiratory way, digestive or physically with anticholinesterases present in pesticides used in the prevention of plagues that affect the crops or in insecticides. These anticholinesterases inhibit the regulatory function of the acetyl-cholinesterase enzyme, which is responsible of metabolizing the acetylcholine (ACh), what derives in an excess of the mentioned neurotransmitter which implies an over excitation of the parasympathetic receptors, and an increase of the frequency of spikes generated by the above mentioned receptors, $f_{t p}$.

Caffeine found in coffee, tea, chocolate, and many soft drinks keeps us awake by counteacting the effects of inhibitory neurotransmitters. Xanthines such as caffeine and theophylline block adenosine receptors [10]. This effect implies an increase in $\alpha$ and $\beta$-sympathetic activity that produces vasoconstriction, higher heart rate and increased heart contraction force. This activity is thought to be responsible for the stimulant effects of these agents [5]. 
The hemorrhage that is defined as an acute blood volume loss (10\% of total or more), modifies directly cardiovascular variables like: systemic arterial pressure, cardiac output and total systemic resistance. In addition, it also affects the heart rate because the system has a baroreceptors feedback also affects the heart rate [13]. A change in total blood volume (positive or negative) as a consequence of transfusion or hemorrhage can be reproduced in Cardiolab.

Panic disorder is a situation with acute autonomic symptoms such as palpitations, difficulty to breathe, heart pounding, tremulousness and fear of dying of a heart attack. Thus, the investigation of abnormal cardiovascular function in panic disorder is of specially interest [15]. In healthy people the Scare could produce a panic crisis or only a light fear, but in all cases the autonomic system switched to an alert situation characterized by higher $\alpha$ and $\beta$ sympathetic activity. In addition in patients with panic disorder there is a decrease in cardiac vagal function proportional to the increase in sympathetic function [15].

\section{The Virtual Laboratory}

\subsection{Tool Development}

Presented software application is based on Easy Java Simulations (EJS) [4], an open source java-based tool that allows creating interactive dynamic simulations.

An EJS application is composed by the three main elements, the model, the view and the interactivity. The model is developed by the designer (transparent for the user) and it can be developed by introducing in EJS a set of differential equations or by using a previous developed simulation model based on another computation tool such as MATLAB/Simulink. As the circulatory model is quite complex (it uses event driven elements with high nonlinearities and the order is high), the simulation model has been implemented in MATLAB/Simulink in order to profit from computation capabilities of this tool. The MATLAB/Simulink can be easily linked to the other elements in order to make interactive the complete systems [9].

The other elements, the view and the interactivity, have been completely designed and implemented in EJS. These elements are the interface to the user, and offer a friendly and interactive way to interact with the laboratory.

\subsection{Interactive Elements}

Interface of the Virtual Laboratory is shown in Fig. 2. The interactive module is on the left side where interesting parameters can be changed by means of sliders and tabs in order to simulate different ventilatory conditions. A multisignal scope can be seen in the right side of the interface when this option is selected by the user. The interactive module is composed by two important types of simulations:
- Cardiovascular stimuli such as exercise, hemorrhage, scare, caffeine and cholinergic intoxication.

- Sympathetic and Parasympathetic gains: $\alpha$ sympathetic gain, $\beta$-sympathetic gain and parasympathetic gain.

In the first one, user can select the kind of stimulus clicking one of the five tabs available. A representative picture is shown in each tab: a kid with his leg bleeding for hemorrhage, a cup of coffee for caffeine, a man fumigating without proper protection for cholinergic intoxication, a victim of a terrible scare during a panic attack and a runner when exercise is simulated as it can be seen in Fig. 2.

User can change the level of stimulus modifying the value of the cardiovascular parameter by means of a slider:

- Hemorrhage: User can simulate a blood lost from zero to $20 \%$ of total blood volume.

- Caffeine: The variable is the volume of drunk coffee, from 0 until 1 liter.

- Cholinergic intoxication: Amount of pesticides in contact with the human body from 0 until $20 \mathrm{mg}$.

- Scare: Subjective scare level, from 0 until 100, being 100 the level that produce a panic attack.

- Exercise: The variable is the exercise work load, from 0 to 100 watts.

Regarding Sympathetic and Parasympathetic panel, the user can stimulate the cardiovascular system with pharmacological products that produce blockade or hyperactivation of sympathetic or parasympathetic nervous systems. In this panel, user can configure its own stimulation profile. Additionally when User sets the stimulus level in the five tabs mentioned above the sympathetic and parasympathetic sliders change their value in agreement and proportionally with the stimulus.

A big animated picture of the heart is shown in the middle of the Virtual Laboratory and its heart period and force of contraction change according to the simulation results. Finally, standard options in Virtual Laboratories are provided such as to "play", to "pause", to "reset", and to save the simulation results ("save-sim"). The last option is available when the simulation is paused and it allows to save the average value of all the variables shown in the Signal Monitor in one MATLAB file (see Fig. 2). It permits further analysis of simulations or comparison with experimental data. Furthermore, external windows appear when the user clicks the options "Show Model" (the MATLAB/Simulink model is shown) and/or "Signal Monitor".

\subsection{Plots and Examples}

One of the two kinds of plots is shown when the corresponding tab of signal scope is selected by the user: Instantaneous and Average. In the former, following variables are shown during the cardiac cycles corresponding 


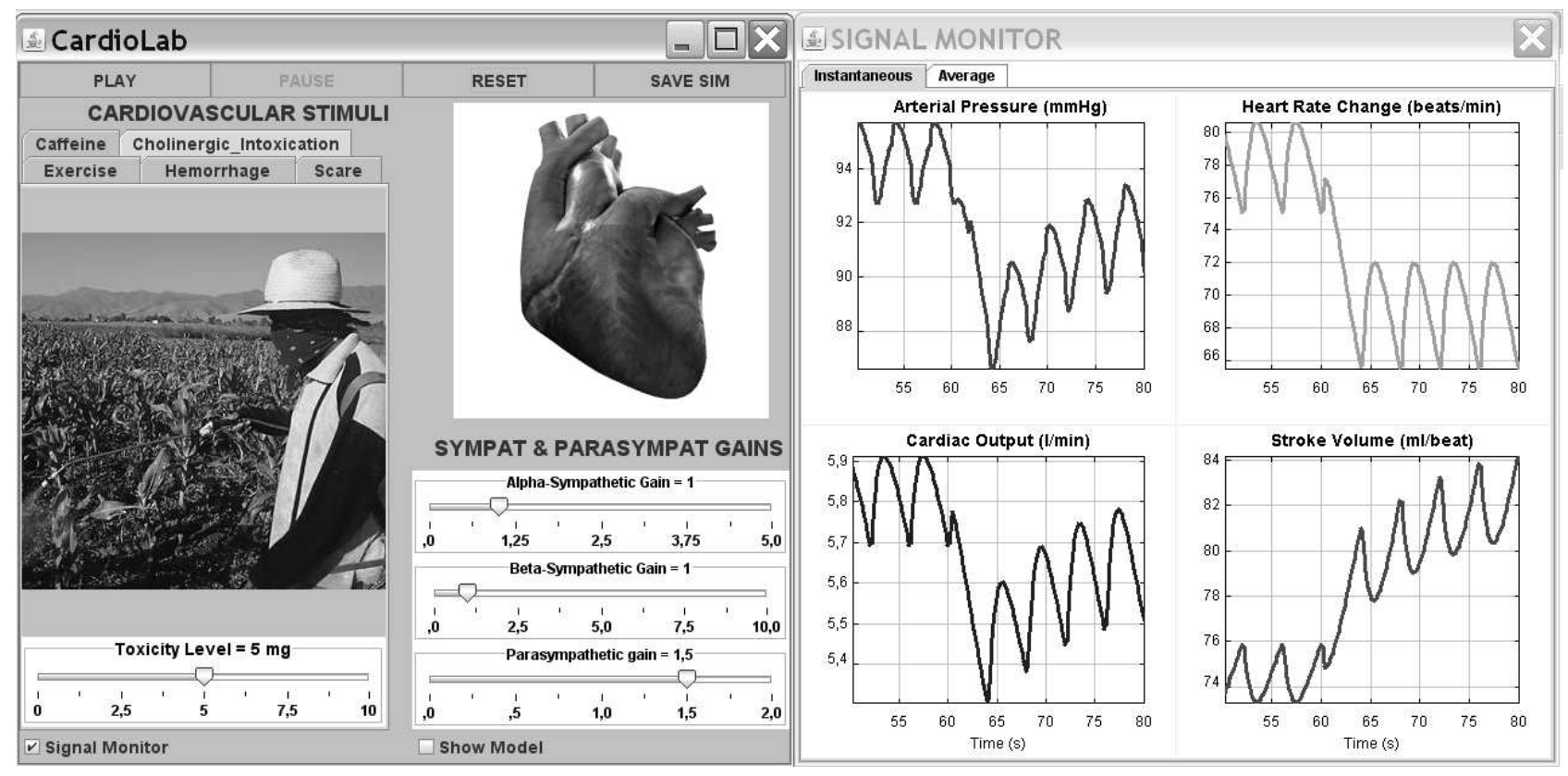

Figure 2. CardioLab Interface. Interface of the CardioLab. Interactive Module and Signals Monitor are shown on the left and right side, respectively.

to the last 30 seconds: arterial blood pressure, heart rate change, cardiac output and stroke volume (see Fig. 2). In the Average option, their values from the beginning of simulation are shown (see 3 ). One illustrative example is used in order to show the capabilities of the tool. It is important to point out that the main characteristic, interactivity, cannot be easily presented in a written text. Nevertheless, several of the useful tools from the Virtual Laboratory are presented below.

Firstly, a change from normal conditions (Toxicity Level $=0 \mathrm{mg}$ ) to a specific amount of pesticides in contact with the human body (Toxicity Level $=5 \mathrm{mg}$ ) is produced at 60 seconds in order to simulate a cholinergic intoxication. As can be seen in Fig. 2, parasympathetic gain increases $50 \%$ due to over excitation of parasympathetic receptors. Instantaneous signals are shown in Fig. 2 where changes in all variables can be observed at that moment. Heart rate goes down because parasympathetic activity increases. On the contrary, the stroke volume goes up because of the unalterable sympathetic activity, and the constant heart force contraction, and longer heart period $(H P=1 / H R)$. This longer heart period and the increased stroke volume produce an underdumped response in arterial blood pressure and cardiac output that are rapidly compensated by the barorreceptors response (see Fig. 2 and 3). After cholinergic intoxication begins, blood pressure and cardiac output remain in a lightly lower value as it can be seen in figure 3 .

The treatment of cholinergic intoxication includes the administration of Atropine because the drug produces a blockade of the parasympathetic system and protects the muscarinic receptors. Based on this fact, a second stimulus is applied at $180 \mathrm{~s}$ : the concentration of pesticides is adjusted to zero and the gain of the parasympathetic system is eliminated in order to produce a blockade (gain = 0 ). The parasympathetic blockade and the elimination of pesticides concentration in the stimuli panel change the heart rate from low to high values and the stroke volume decreases because heart force contraction remains unalterable and $\alpha$ and $\beta$-sympathetic gains do not change with the drug.

It is important to note that cardiac output is the product of the stroke volume and heart rate. Reduced heart period and stroke volume produce an underdumped response in arterial blood pressure and cardiac output that are rapidly compensated by the barorreceptors response as in it is shown in Fig. 3. After Atropine administration, blood pressure and cardiac output remain in higher values as it can be seen in Fig. 3 .

\section{Conclusions}

In this paper a virtual laboratory designed to analyze the human circulatory control system has been introduced. This laboratory is completely graphic and interactive, so it can be used to illustrate the behavior of human circulatory control system under certain circumstances and the influence of relevant parameters in the system. This virtual lab allows the students and researchers obtain sensations and experience that would be very difficult otherwise because of the difficulties in performing experimental human studies. The use of virtual laboratories and interac- 


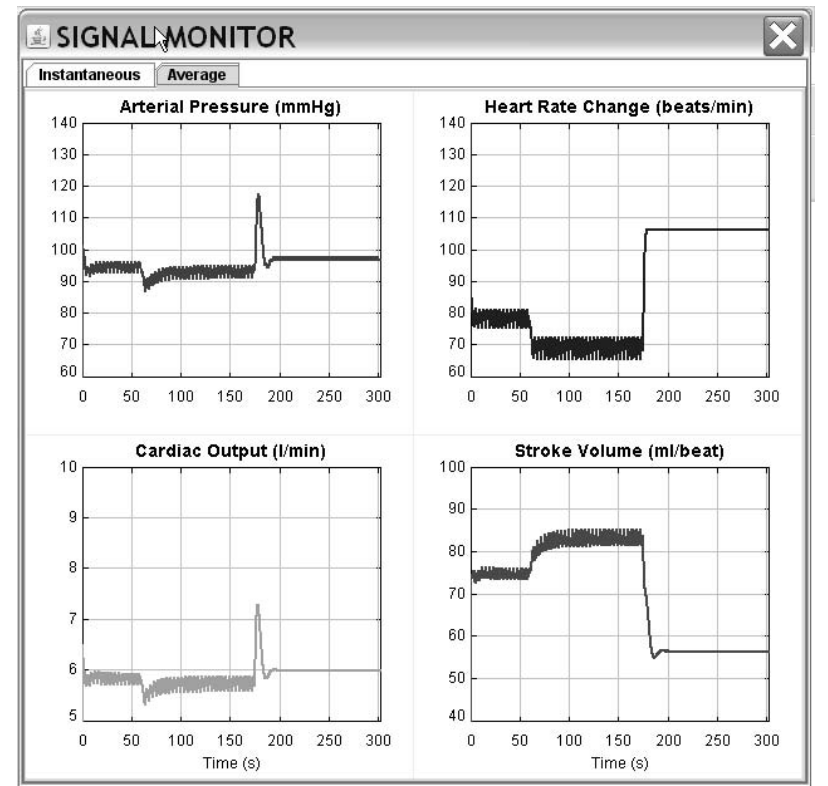

Figure 3. Multisignal scope for average values.

tivity in BME has proved to be an efficient way to shortcut the learning process an improve the students capabilities.

The tool has been built combining MATLAB/Simulink and EJS. While MATLAB/Simulink allows to implement complex models in straightforward manner, EJS allows to design attractive views and introduce interactivity easily. This combination is quite suitable for virtual lab. development.

\section{References}

[1] J. D. Bronzino. The Biomedical Engineering Handbook. CRC Press, Inc., 2000.

[2] S. Cavalcanti and E. Belardinelli. Modeling of cardiovascular variability using a differential delay equation. IEEE Trans Biomed Eng, 43(10), October 1996.

[3] S. Dormido, S. Dormido, R. Dormido, J. Sánchez, and N. Duro. The role of interactivity in control learning. International Journal of Engineering Education, 21(6):1122-1133, 2005.

[4] F. Esquembre. Creacion de simulaciones interactivas en Java. Pearson Educación - Prentice Hall, 2005.

[5] B. B. Fredholm. Adenosine, adenosine receptors and the actions of caffeine. Pharmacol. Toxicol, 76:93-101, 1995.

[6] J. Freeman, F. Dewey, D. Hadley, J. Myers, and V. Froelicher. Autonomic nervous system interaction with the cardiovascular system during exercise. Progress in Cardiovascular Diseases, 48(5):342-362, 2006.

[7] O. Ivanova and M. Khoo. Simulation of spontaneous cardiovascular variability using pneuma. In 26th Annual International Conference of the IEEE Engineering in Medicine and Biology Society (IEMBS '04)., volume 2, pages 3901-3904, September 2004.

[8] J. Sánchez, S. Dormido, and F. Esquembre. The learning of control concepts using interactive tools. Computer Applications in Engineering Education, 13(1):84-98, 2004.
[9] J. Sánchez, F. Esquembre, C. Martín, S. Dormido, R. Dormido, S. Dormido, and R. Pastor. Easy java simulations: an open-source tool to develop interactive virtual laboratories using matlab/simulink. International Journal Engineering Education, 21(5):798-813, 2005.

[10] J. C. Shryock and L. Belardinelli. Adenosine and adenosine receptors in the cardiovascular system: Biochemistry, physiology, and pharmacology. The American Journal of Cardiology, 79(12):2-10, June 1997.

[11] B. TenVOORDE, T. Faes, T. Janssen, G. Scheffer, and O. Rompelman. Respiratory modulation of blood pressure and heart rate studies with a computer model of baroreflex control, chapter Computer analysis of cardiovascular signals. IOS pressure, 1995.

[12] H. Tsuchimochi, K. Matsukawa, H. Komine, and J. Murata. Direct measurement of cardiac sympathetic efferent nerve activity during dynamic exercise. Am J Physiol Heart Circ Physiol, 283(5):1896-1906, 2002.

[13] M. Ursino. Interaction between carotid baroregulation and the pulsating heart: a mathematical model. Am. J. Physiol, 44:1733-1747, 1998.

[14] M. Ursino and E. Magosso. Role of short-term cardiovascular regulation in heart period variability: a modeling study. Am J Physiol Heart Circ Physiol, 284(4):1479_ 1493, 2003.

[15] V. K. Yeragani, M. Tancer, and T. Uhde. Heart rate and qt interval variability: abnormal alpha- 2 adrenergic function in patients with panic disorder. Psychiatry Research, 121(2):185-196, December 2003. 